\title{
The Facilitated Processing of Threatening Faces: An ERP Analysis
}

\author{
Harald T. Schupp \\ University of Greifswald and University of Konstanz \\ Markus Junghöfer \\ University of Konstanz
}

\author{
Arne Öhman \\ Karolinska Institute
}

\author{
Almut I. Weike, Jessica Stockburger, and \\ Alfons O. Hamm \\ University of Greifswald
}

\begin{abstract}
Threatening, friendly, and neutral faces were presented to test the hypothesis of the facilitated perceptual processing of threatening faces. Dense sensor event-related brain potentials were measured while subjects viewed facial stimuli. Subjects had no explicit task for emotional categorization of the faces. Assessing early perceptual stimulus processing, threatening faces elicited an early posterior negativity compared with nonthreatening neutral or friendly expressions. Moreover, at later stages of stimulus processing, facial threat also elicited augmented late positive potentials relative to the other facial expressions, indicating the more elaborate perceptual analysis of these stimuli. Taken together, these data demonstrate the facilitated perceptual processing of threatening faces. Results are discussed within the context of an evolved module of fear (A. Öhman \& S. Mineka, 2001).
\end{abstract}

The human face displays a wealth of psychologically relevant information. For instance, gender, age, and visual identity can be rapidly extracted from cranial and facial appearances of the face. Furthermore, and even more important for the present context, faces also supply information regarding the attentional focus and motivational and emotional states. Thus, facial expressions are central to nonverbal social exchange as markers of internal states and as signals of intentions. This dual role of facial expressions is

Harald T. Schupp, Department of Psychology, University of Greifswald, Greifswald, Germany, and Department of Psychology, University of Konstanz, Konstanz, Germany; Arne Öhman, Department of Clinical Neuroscience, Karolinska Institute, Stockholm, Sweden; Markus Junghöfer, Department of Psychology, University of Konstanz; Almut I. Weike, Jessica Stockburger, and Alfons O. Hamm, Department of Psychology, University of Greifswald.

This research was supported by German Research Foundation Grants Schu1074/7-1 awarded to Harald T. Schupp and Ha1593/10-2 awarded to Alfons O. Hamm. We thank Johannes Bahlmann for his assistance in data collection.

Correspondence concerning this article should be addressed to Harald T. Schupp, who is now at the Institute of Psychology, University of Konstanz, P.O. Box D36 78457, Konstanz, Germany. E-mail: harald.schupp@uni-konstanz.de readily illustrated by considering anger expressions. Whereas angry expressions are characterized by frowning brows, starring eyes, and a shut mouth with tense lips (Ekman \& Friesen, 1975), these expressions also signal a readiness for physical or symbolic attack for the observer of such a face. The present study focused on the signaling rather than the expressive role of facial expressions, and therefore, these faces are referred to as threatening rather than angry faces. For similar reasons, happy expressions are referred to as friendly faces (cf. Öhman, Lundqvist, \& Esteves, 2001). Considered from an evolutionary perspective, Öhman (1986) proposed that threatening faces are prototypical for activating the human fear system. Consistent with this notion, fear-relevant threatening faces demonstrate superior fear conditioning compared with friendly faces (reviewed in Öhman \& Mineka, 2001). However, although escape and avoidance are the essential response characteristics of the fear system, the successful avoidance of threat requires the efficient analysis of the environment in order to locate cues suggesting impending doom. Consistent with this hypothesis, recent studies demonstrated an advantage to detect threatening compared with friendly schematic faces (Öhman, Lundqvist, \& Esteves, 2001). The present study aimed to provide further evidence for the facilitated processing of 
threatening stimuli. Specifically, event-related brain potential components (ERPs) targeting perceptual stimulus encoding were used to examine the facilitated processing of threatening faces.

The hypothesis that threatening (schematic) faces are detected more quickly than nonthreatening faces has been studied recently with visual search tasks (Fox et al., 2000; Öhman, Lundqvist, \& Esteves, 2001; Tipples, Atkinson, \& Young, 2002). For instance, in a series of studies (Öhman, Lundqvist, \& Esteves, 2001), subjects searched for discrepant faces in matrices of otherwise identical faces. Across experiments, threatening faces were detected more quickly than friendly faces among both neutral and emotional distractors. Furthermore, the effect extended to inverted threatening faces and was also observed in comparison with other negative expressions (sad faces). Replicating the threat advantage, Tipples et al. (2002) showed that the eyebrow frown is particularly implicated in the quick detection of threatening faces. Furthermore, Byrne and Eysenck (1995) demonstrated that highly anxious individuals were faster than low-anxious volunteers in detecting threat among neutral real faces in a visual search paradigm. In addition, high-anxious subjects were more distracted by threatening faces serving as background stimuli. Conceptually similar findings have been reported for individuals with generalized social phobia (Gilboa-Schechtman, Foa, \& Amir, 1999). Further evidence for the increased allocation of attention to threat faces is provided by studies using the modified emotional Stroop and dot probe paradigms (e.g., Mogg, Millar, \& Bradley, 2000).

Although these data provide behavioral evidence that threatening faces capture attentional resources, a processing advantage of threatening faces has also been demonstrated by psychophysiological measures. For instance, Öhman and Dimberg (1978) used classical conditioning to compare threatening faces with pictures of neutral or friendly faces as conditioned stimuli, followed by an aversive unconditioned shock stimulus. Reliable acquisition of conditioned skin conductance responses could be observed for threatening, neutral, and friendly expressions; however, only threatening faces demonstrated conditioned responses during extinction. More recent studies indicate that the conditioning advantage of threatening faces can be observed when the faces are presented outside of awareness, either during acquisition (Esteves, Parra, Dimberg, \& Öhman, 1994) or during extinction (Esteves, Dimberg, \& Öhman, 1994). Furthermore, Dimberg and Thunberg (1998) observed differences in specific facial muscles when subjects viewed expressions of anger and happiness. Again, these differences were confirmed under conditions of backward masking preventing conscious recognition of the stimuli (Dimberg, Elmehed, \& Thunberg, 2000).

Öhman (1986) suggested that threatening faces are powerful stimuli to activate the fear system because they are fear relevant in an evolutionary perspective. Considered from an evolutionary-based categorization of behaviors, threatening faces might be considered more specifically as prototypical fear stimuli of an evolved behavioral system related to conspecific attack and self-defense. The system controls the interaction among individuals in a group by defining dominance and social submissiveness among individuals. Thus, the social submissiveness system serves to avert attack and humiliation from social conspecifics, thereby avoiding physical and mental harm. However, a further evolutionary-derived behavioral system reflects the need for effective predatory defense, presumably explaining the prominent role of animal fears in humans. Interestingly, using stimuli associated with the predatory fear system, research also demonstrated the processing advantage of fearrelevant stimuli in visual search and associative conditioning paradigms (cf. Öhman \& Mineka, 2001). For instance, researchers found that pictures of snakes and spiders were detected faster in grid-pattern arrays of fear-irrelevant pictures (flowers and mushrooms) than vice versa. Furthermore, this effect was more pronounced for subjects with specific fears of these animals (Öhman, Flykt, \& Esteves, 2001). Independent evidence from neuroimaging studies support the hypothesis of facilitated encoding at the level of visual stimulus analysis. For instance, patients with snake phobias showed enlarged activity in the visual associative cortex while viewing fear-relevant films (Fredrikson et al., 1993).

Pursuing a neuroscientific perspective, the present study was designed to provide evidence for the facilitated perceptual encoding of threatening faces. ERPs can serve this purpose because this measure provides information about stimulus encoding with a millisecond resolution. Previous research demonstrated two ERP components of facilitated emotional stimulus encoding. Presenting emotional and neutral images from the International Affective Picture System (IAPS; Lang, Bradley, \& Cuthbert, 1999), we recently observed that the early posterior negativity (EPN) was the first cortical ERP component reflecting the facilitated processing of emotional stimuli. The EPN com- 
ponent developed around $150 \mathrm{~ms}$ and was maximally pronounced around 260-280 ms after picture onset. In addition, the amplitude of the EPN was most pronounced for stimuli of high evolutionary significance, that is, erotic images and pictures of mutilations (Junghöfer, Bradley, Elbert, \& Lang, 2001; Schupp, Junghöfer, Weike, \& Hamm, 2003b). Source analysis of the EPN amplitude implicated a widespread network of temporo-parieto-occipital areas implicated in visual information processing (Junghöfer et al., 2001). Furthermore, a recent functional magnetic resonance imaging (fMRI) study using rapid visual picture presentation (Junghöfer et al., 2002) revealed increased activations by emotional pictures in occipital (occipital, lingual and fusiform gyrus, cuneus, calcarine), temporal (superior, mid- and inferior-temporal gyrus), and parietal (inferior and superior parietal, angular, supramarginal gyrus, precuneus) structures. Thus, the EPN component presumably reflects the processing at the early perceptual level.

However, the facilitated processing of emotional pictures was also reflected at a later time of stimulus processing. Specifically, emotionally arousing (pleasant and unpleasant) pictures elicited enlarged late positive potentials (LPPs) over centro-parietal sensors developing around 350-400 ms after stimulus onset and lasting for several hundred milliseconds (e.g., Cuthbert, Schupp, Bradley, Birbaumer, \& Lang, 2000; Schupp et al., 2000; Schupp et al., in press; Schupp et al., 2003b). Again, this phenomenon was related to the intensity of the emotional stimulation, that is, more pronounced for unpleasant and pleasant pictures of high emotional arousal (Cuthbert et al., 2000; Schupp et al., 2000; Schupp et al., 2003b). In cognitive research, researchers found that LPPs reflect further processing related to the perceptual demands of the task after the initial stimulus identification is completed (e.g., Ritter \& Ruchkin, 1992). According to these findings, it was reasoned that the intrinsic relevance of emotional stimuli is reflected by more elaborate perceptual analysis of these stimuli (cf. Cuthbert et al., 2000).

Building on these findings, the facilitated perceptual processing of threatening faces was explored in the present study by two specific ERP components targeting the early and late perceptual processing. However, to incorporate more ecologically valid stimulus materials than the previously used schematic faces (cf. Tipples et al., 2002), in the present study subjects viewed human faces depicting threatening, friendly, and neutral expressions, which were selected from the Karolinska Directed Emotional Faces series
(KDEF; Lundqvist, Flykt, \& Öhman, 1998). Importantly, a large number of individual expressions could be presented, and each individual posed all emotional displays. Thus, images were controlled for physical characteristics (e.g., background, dress, color, and so forth), and idiosyncrasies of particular individuals contributed equally to all experimental conditions (cf. Haxby, Hoffman, \& Gobbini, 2000). Furthermore, measuring ERPs enables the assessment of the facilitated processing of threat pictures implicitly-that is, in the absence of any task or instruction for evaluative categorization of the expressions. Thus, the present study enabled the demonstration of facilitated processing of threatening faces, whereas subjects did not invoke specific attentional control settings (Folk, Remington, \& Johnston, 1992). A further goal of the present study was to provide more direct evidence for the facilitated perceptual processing of threatening faces. In principle, the efficient detection of threatening faces suggested by previous studies might be due to facilitated perceptual encoding, a shift in decision criteria, or response mobilization (cf. Luck, Woodman, \& Vogel, 2000; Mangun \& Hillyard, 1991). Thus, acknowledging the need to distinguish between the various stages of information processing (cf. Cacioppo, Gardner, \& Berntson, 1999; Luck et al., 2000), the present measure specifically isolates perceptual processing. On the basis of previous findings on emotional stimulus processing, we predicted that threatening pictures would modulate two specific ERP components: (a) Focusing on the early perceptual level of stimulus analysis, we predicted that threatening faces would be associated with enlarged EPN amplitudes compared with friendly and neutral faces; and (b) similarly, focusing on the continued perceptual processing, we expected that threatening facial expressions would be associated with augmented amplitudes of the LPP. Furthermore, aversive conditioning studies showed an advantage of threatening faces only when the threat faces were directed toward the subjects (Dimberg \& Öhman, 1983). Thus, the present study presented the faces directed either toward the subjects or turned halfway toward the right or left in order to examine whether these effects are also obtained with measures of stimulus encoding.

\section{Method}

\section{Subjects}

Subjects were 20 introductory psychology students (10 women) from the University of Greifswald, Greifswald, Germany, who received course credits 
toward their research requirements. One subject had to be excluded from data analyses because of excessive eye movement artifacts. Subjects were between the ages of 19 and 31 years $(M=23.2)$.

\section{Stimulus Materials}

Facial expressions were selected from the KDEF (Lundqvist et al., 1998). The KDEF consists of posed facial expressions (happiness, surprise, fear, disgust, sadness, anger, neutral) by actors in training who received written instructions of each emotional expression and were actively coached to produce the appropriate expression according to Ekman and Friesen (1975). Threatening (angry), friendly (happy), and neutral expressions were selected from 33 individuals (17 men) posing the expression in frontal orientation, and mid-left and mid-right orientations $\left(\sim 45^{\circ}\right)$ were considered from the perspective of the viewing person.

\section{Pilot Study: Subjective Ratings of the Facial Expressions}

Recent studies using schematic face stimuli indicated that the eyebrow region is particularly relevant for categorizing these schematic faces as threatening or nonthreatening. Specifically, expressions displaying "frowning" $\vee$-shaped eyebrow regions were rated as more negative and arousing compared with friendly faces (Lundqvist, Esteves, \& Öhman, 1999; Tipples et al., 2002). Similar results were obtained for photographic face stimuli from the Ekman series (Johnsen, Thayer, \& Hugdahl, 1995). Specifically, threatening faces were perceived as more unpleasant and more arousing than friendly or neutral faces. However, other researchers observed rather increased intensity of expression for happy compared with angry expressions (Kesler/West et al., 2001). Therefore, a pilot study was conducted in which 14 introductory psychology students ( 8 women) were asked to rate the facial expressions in terms of how threatening, pleasant, and arousing the expressions appeared. A 9-point line rating scale was used to assess the threat dimension, while the Self-Assessment Manikin (Lang, 1980) served to assess valence and arousal (Bradley \& Lang, 1994). Facial stimuli were presented in a perceptually random order. Threat, valence, and arousal ratings were subjected to separate repeated-measures analyses of variance (ANOVAs), with emotion (threat vs. neutral vs. friendly) and orientation (mid-left vs. frontal vs. mid-right) as between-subjects factors. Table 1 summarizes the subjective ratings of the facial expressions. As expected, threatening faces were per-
Table 1

Mean Threat, Valence, and Arousal Ratings When Viewing Friendly, Neutral, and Threatening Faces in Mid-Left, Frontal, and Mid-Right Orientations

\begin{tabular}{|c|c|c|c|c|c|c|}
\hline \multirow[b]{2}{*}{ Dependent measure } & \multicolumn{2}{|c|}{ Friendly } & \multicolumn{2}{|c|}{ Neutral } & \multicolumn{2}{|c|}{ Threatening } \\
\hline & $M$ & $S D$ & $M$ & $S D$ & $M$ & $S D$ \\
\hline \multicolumn{7}{|c|}{ Threat ratings (1-9 scale) } \\
\hline Mid-left & 1.6 & 0.5 & 2.7 & 1.3 & 5.0 & 1.5 \\
\hline Frontal & 1.7 & 0.6 & 3.1 & 1.1 & 5.6 & 1.4 \\
\hline Mid-right & 1.7 & 0.6 & 3.1 & 1.0 & 5.2 & 1.4 \\
\hline \multicolumn{7}{|c|}{ Pleasure ratings (1-9 scale) } \\
\hline Mid-left & 7.2 & 0.6 & 5.5 & 0.9 & 3.7 & 0.7 \\
\hline Frontal & 7.2 & 0.8 & 5.1 & 0.9 & 3.4 & 0.7 \\
\hline Mid-right & 7.2 & 0.7 & 5.5 & 0.9 & 3.6 & 0.8 \\
\hline \multicolumn{7}{|c|}{ Arousal ratings (1-9 scale) } \\
\hline Mid-left & 2.8 & 1.0 & 2.9 & 1.0 & 4.9 & 1.8 \\
\hline Frontal & 3.1 & 1.1 & 3.3 & 1.0 & 5.2 & 1.8 \\
\hline Mid-right & 2.8 & 1.1 & 2.9 & 1.0 & 5.0 & 1.7 \\
\hline
\end{tabular}

Note. Facial orientation is indicated from the perspective of the observer.

ceived as more threatening than either neutral or friendly faces (emotion), $F(2,26)=65.8, p<.0001$ $(\varepsilon=0.765)$; contrast $F \mathrm{~s}(1,13)>69, p<.0001$. Furthermore, neutral expressions were perceived as more threatening than friendly faces, $F(1,13)=20.6$, $p<.001$. Similar results were obtained for pleasantness ratings of the expressions (emotion), $F(2,26)=$ 92.2, $p<.0001(\varepsilon=0.736)$. Threatening faces were rated as more unpleasant than neutral faces, which were less pleasant than friendly faces, contrast $F \mathrm{~s}(1$, 13) $>42, p<.0001$. In addition, the facial expressions were also associated with different arousal ratings (emotion), $F(2,26)=24.4, p<.0001(\varepsilon=0.761)$. Threatening faces were perceived as more arousing than either friendly or neutral faces, which did not differ from each other, contrast $F_{\mathrm{s}}(1,13)>25, p<$ .0001 . Significant differences also emerged with respect to the orientation of the facial expressions for each of the three rating variables (orientation), $F \mathrm{~s}(2$, $26)=14.0,5.6$, and 8.6, $p<.05$, for threat, valence, and arousal, respectively; Orientation $\times$ Emotion, $F \mathrm{~s}(2,26)=3.9$ and $3.6, p<.05$, for threat and valence, respectively. However, these differences were rather small in size (cf. Table 1) and demonstrated in general higher emotionality for faces presented frontally.

\section{Procedure}

The total of 297 facial stimuli were presented in a perceptually random order according to facial expression and facial orientation, and no more than two repetitions of the same expression (threat, neutral, 
friendly) or direction (frontal, mid-left, mid-right) were allowed. Faces were displayed for 1,000 ms, and a warning dot $(500 \mathrm{~ms})$ preceded picture onset to ensure that subjects were fixating on the center of the screen. The intertrial interval varied between 1,750 and 2,250 ms and was 2,000 ms on average. Each subject received his or her own order of picture presentation. Three pictures served as practice trials. Subjects were instructed to simply view the pictures without any further instruction, that is, to pay attention to the emotion or orientation of the faces.

\section{Apparatus and Data Analysis}

Electrophysiological data were collected from the scalp using a 129-channel system (Electrical Geodesics, Inc., Eugene, OR). Scalp impedance for each sensor was kept below $30 \mathrm{k} \Omega$, which is appropriate for this type of amplifier. The electroencephalogram (EEG) was recorded continuously with a sampling rate of $250 \mathrm{~Hz}$, the vertex sensor as reference electrode, and online bandpass filtered from .01 to 100 Hz. Continuous EEG data were low-pass filtered at $35 \mathrm{~Hz}$ using digital filtering before stimulus synchronized epochs were extracted from $200 \mathrm{~ms}$ before until $800 \mathrm{~ms}$ after picture onset. The raw EEG epochs were passed through a computerized artifact detection algorithm. Trials with blink artifacts and on which 10 or more channels exceeded a voltage threshold of $200 \mu \mathrm{V}$ (absolute) or a transition threshold of $100 \mu \mathrm{V}$ (sample to sample) were excluded from the calculation of the final average. On average, $8.2 \%$ of the trials were excluded from analysis, similarly distributed across conditions. Thus, trials in which only a few channels were out of range, indicating a focal signal problem, were retained with the contaminated sensors excluded for that trial only. Finally, data were transformed to an average reference to reduce bias resulting from the choice of reference sensor.

Separate average waveforms were calculated for the nine experimental cells (friendly, neutral, and threatening face; and mid-left, frontal, and mid-right orientation) for each sensor and subject. A two-step procedure was used to analyze the threat-related modulation of the ERP waveform. First, nonparametric Wilcoxon's signed-rank tests (threat vs. neutral faces and threat vs. friendly faces) were calculated for each time point after picture onset separately for each individual sensor to identify the temporal and spatial characteristics of the facilitated processing of threatening faces. These waveform analyses were conducted using a significance criterion of $p<.01$. To avoid false positives, significant effects were only considered meaningful when the effects were observed for at least eight continuous data points (32 $\mathrm{ms})$ and two neighboring sensors revealing significant threat-related modulation. In a second step, the outcome of these waveform analyses served to collapse information according to the spatial focus and temporal characteristics of the affective modulation. Regions of interest were defined, and the average of the selected sensors was calculated. Similarly, the mean activity was obtained within critical time windows. These data were analyzed by repeated-measures ANOVAs to provide a more conventional ERP analysis and brevity of result presentation.

No reliable modulation of the ERP waveform was observed in the first $200 \mathrm{~ms}$ after stimulus onset. In particular, the N170 component, which is related to the structural encoding of faces, was not reliably modulated by the emotional display of the facial expressions (cf. Bentin, Allison, Puce, Perez, \& McCarthy, 1996). However, the waveform analysis indicated significant modulations for the EPN and LPP components.

EPN. Augmented EPN amplitudes to threatening faces emerged around $200 \mathrm{~ms}$ and lasted for approximately $120 \mathrm{~ms}$. As in previous studies (Schupp et al., 2003b), this modulation was observed in bilateral clusters of enlarged relative temporo-occipital negativity and corresponding centro-medial positivity for threatening compared with other facial stimuli. The early facilitated processing is referred to as a relative negative shift in the potential waveform over temporo-occipital sites because the facilitated processing of emotional cues appears uniformly as negative shift in the waveform despite the various appearances of the evoked visual potential waveforms for other experimental designs or stimulus materials (Junghöfer et al., 2001; Schupp et al., 2003b). Accordingly, the EPN amplitude was analyzed with a repeatedmeasures ANOVA, with emotion (threat vs. neutral vs. friendly), orientation (mid-left vs. frontal vs. midright), and laterality (left vs. right) as betweensubjects factors. The EPN amplitude was scored as mean activity in the time interval from 240 to $280 \mathrm{~ms}$ collapsed over sensors with Electrical Geodesic (Electrical Geodesic, Inc., Eugene, OR) Sensor Numbers $56,63,64,65,66,69,70,71,74$, and 75 on the left, and Sensor Numbers 83, 84, 85, 89, 90, 91, 95, 96, 100 , and 108 on the right hemisphere. For brevity, this ANOVA analysis focused on the temporo-occipital sites because the effects at centromedial sites mirrored the posterior effects. 


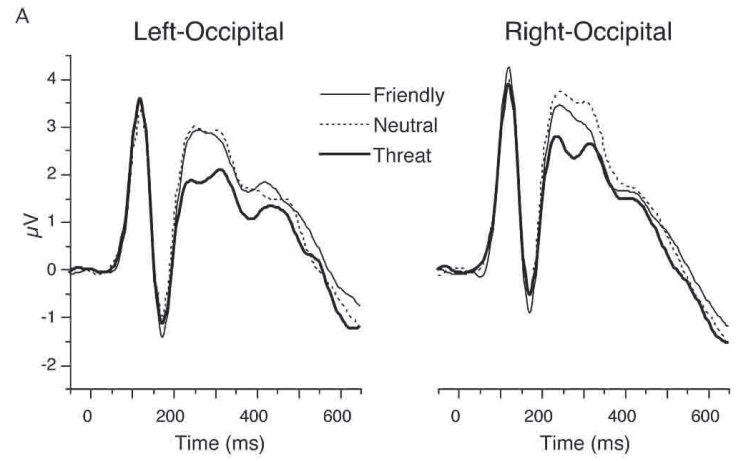

B

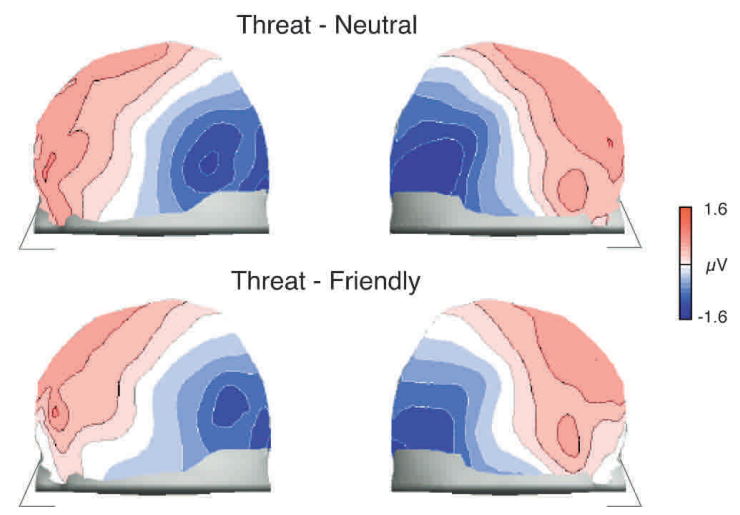

Figure 1. A: Grand-averaged event-related potential waveforms during viewing of threat, neutral, and friendly faces. Left and right hemispheric sensors were selected for representing the early posterior negativity over the temporooccipital sites (Sensors 65 and 91) elicited by threatening faces. B: Scalp potential maps reveal the topography of the early facilitated processing of threatening faces. Bilateral foci of temporo-occipital negativity appeared for threatening compared with friendly and neutral expressions (with a corresponding polarity reversal over centromedial sites). Illustrated is a left and right side view of the model head.

$L P P$. A second component reflecting significant threat-related modulation were augmented LPP amplitudes over centro-parietal sensors developing around $400 \mathrm{~ms}$ and lasting for several hundred milliseconds. Accordingly, an ANOVA, with emotion, orientation, and laterality as between-subjects factors, was calculated for the LPP. The LPP was scored as mean activity in the time interval from 400 to $500 \mathrm{~ms}$, averaged over centro-parietal Sensor Numbers 32, 38, $43,48,52,53,54,60,61$, and 79, 80, 81, 86, 87, 88, 93, 94, 99 on the left and right hemispheric sites, respectively.

For effects involving repeated measures, the Greenhouse-Geisser epsilon was used to correct for violations of sphericity.

\section{Results}

As illustrated in Figures 1 and 2, the present study obtained evidence for the facilitated processing of threatening faces, as indexed by both the EPN and LPP amplitudes. Augmented EPN amplitudes over temporo-occipital sensors to threatening compared with friendly and neutral faces developed around 200 $\mathrm{ms}$ after stimulus onset and were most pronounced around $280 \mathrm{~ms}$ poststimulus (see Figure 1A). Thus, the EPN modulation appeared as a relative negative polarity shift over temporo-occipital sensors, whereas the overall ERP waveform presented a positive polarity over posterior sensors. Furthermore, appearing around $400 \mathrm{~ms}$ and lasting for around $200 \mathrm{~ms}$, threat stimuli were associated with enlarged LPP amplitudes at centro-parietal sensors (see Figure 2A). Information about the temporal development of facilitated threat encoding is complemented by illustration of the spatial topography of the EPN and LPP. As shown in

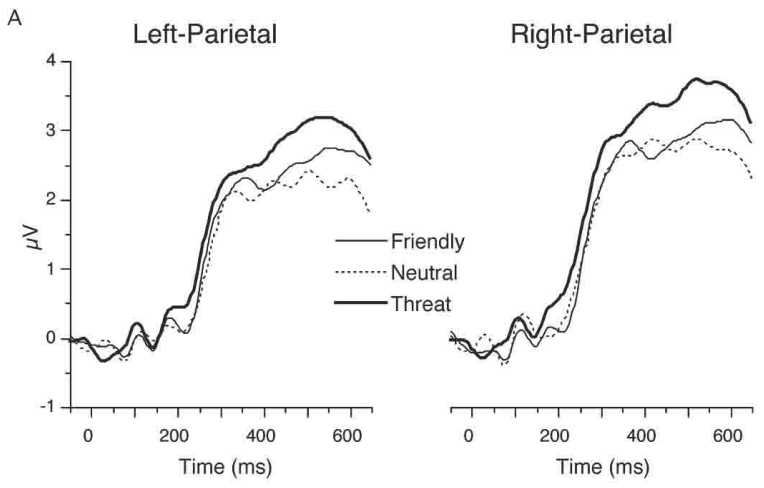

B
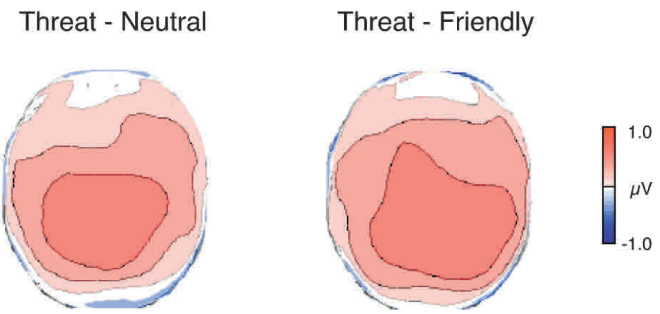

Figure 2. A: Grand-averaged event-related potential waveforms during viewing of emotional expressions. A representative left and right hemispheric sensor (Sensors 53 and 87) was selected for representing the late positive potential (LPP) observed over centro-parietal sites. B: Scalp potential maps reveal the topography of the late facilitated processing of threatening faces. Augmented LPP amplitudes were observed for threatening compared with friendly and neutral expressions, with a broad centro-parietal topography. Illustrated is a top view of the model head. 
Figure 1B, the calculation of difference maps (threat minus neutral, threat minus friendly) revealed bilateral sources of increased temporo-occipital negativity for threat faces compared with friendly and neutral expressions. Furthermore, Figure 2B illustrates the broad centro-parietal topography of augmented LPP amplitudes to threatening faces. These descriptive findings were supported by the statistical analyses of individual waveforms and by conventional ANOVA statistics.

\section{EPN}

Threatening expressions elicited significantly larger negativity over temporo-occipital sites compared with neutral and friendly faces (emotion), $F(2$, $36)=17.3, p<.0001(\varepsilon=0.94)$; contrast $F \mathrm{~s}(1,18)$ $=27.6$ and 18.2, $p<.0001$. This effect was not modulated by the orientation of the faces, Emotion $x$ Orientation, $F(4,72)<1$. In addition, separate analyses of the three orientations confirmed the significance of these findings. That is, increased posterior negativity for threat faces was similarly observed for mid-left, frontal, and mid-right orientations, $F \mathrm{~s}(1,18)$ $>10.5, p<.01$, for threat versus neutral; and $F \mathrm{~s}(1,18)$ $>6.0, p<.05$, for threat versus friendly expressions, respectively.

In general, facial stimuli elicited more negative potentials for frontal orientations compared with midleft or mid-right orientations across all expressions (orientation), $F(2,36)=7.1, p<.01(\varepsilon=0.89)$; contrast $F \mathrm{~s}(1,18)>9, p<.01$. Furthermore, as indicated by a significant Orientation $\times$ Laterality interaction, $F(2,36)=8.4, p<.01(\varepsilon=0.80)$, frontal and mid-right oriented expressions elicited more negative potentials over left compared with right temporooccipital sensors, $F \mathrm{~s}(1,18)>4.7, p<.05$.

\section{$L P P$}

The facilitated encoding of threatening faces was also observed for the LPP (emotion), $F(2,36)=10.0$, $p<.001(\varepsilon=0.99)$. Threatening faces elicited augmented LPP amplitudes over centro-parietal sensors compared with nonthreatening neutral and friendly displays, $F \mathrm{~s}(1,18)=17.4$ and $11.7, p<.01$. Although inspection of the data revealed that the facilitated processing of threat faces was primarily observed for frontal and mid-right orientations, this Emotion $\times$ Orientation interaction failed to reach statistical significance, $F(4,72)=1.6, p=.18(\varepsilon=$ 0.71).

Furthermore, analogous to the early ERP component, frontal and mid-right oriented expressions elic- ited somewhat more positive potentials over right than left parietal sensors, $F_{\mathrm{S}}(1,18)>4.7, p<.05$; Orientation $\times$ Laterality, $F(2,36)=5.5, p<.05(\varepsilon=$ $0.85)$.

\section{Discussion}

An evolutionary/functional analysis of the fear system suggests that escape and avoidance are the central characteristics of the fear system. However, because of the flexible tailoring of responses to environmental contingencies in humans, successful avoidance of threatening situations depends on the accurate perceptual encoding of the environment (Öhman, Flykt, \& Esteves, 2001; Öhman, Flykt, \& Lundqvist, 2000). Consistent with this hypothesis, visual search studies suggest faster detection of threatening compared with friendly faces (Öhman, Lundqvist, \& Esteves, 2001; Tipples et al., 2002). To provide more direct evidence for the facilitated perceptual processing of threatening faces, the present study measured ERPs. Assessing early perceptual stimulus processing, threatening faces elicited enlarged EPN amplitudes compared with neutral or friendly expressions. Moreover, at later stages of stimulus processing, facial threat also elicited augmented LPPs relative to the other facial expressions. Taken together, these data demonstrate the facilitated perceptual processing of threatening faces.

Consistent with behaviorally based theories of face recognition that postulate independent processing of identity, expression, and visually perceivable speech movements of faces (e.g., Bruce \& Young, 1986), neurophysiological and neuroimaging data suggest that facial stimuli are processed in a distributed neural system (e.g., Allison, Puce, \& McCarthy, 2000; Haxby et al., 2000). For instance, the fusiform gyrus and adjacent inferior temporal and occipital gyri are particularly implicated in face perception and recognition, whereas the superior temporal sulcus (STS) appears to be particularly important for the processing of dynamic features of faces such as emotional expression and gaze direction. The first negative deflection in the ERP waveform, the N170, reflects the early visual processing responsible for a sensory representation of faces prior to face recognition (Bentin et al., 1996). However, the N170 component was not modulated by the emotional content of the faces. This concurs with studies exploring the processing of fearful compared with neutral faces (e.g., Holmes, Vuilleumier, \& Eimer, 2003; Sato, Kochiyama, Yoshikawa, \& Matsumura, 2001). Interestingly, instructing sub- 
jects to direct attention explicitly to faces did not modulate the N170 (Cauquil, Edmonds, \& Taylor, 2000), although spatial attention tasks may enlarge the N170 (Holmes et al., 2003). Considering the reliable modulation of the fusiform area by fearful faces and emotional pictures in fMRI studies (e.g., Bradley et al., 2003; Pessoa, Kastner, \& Ungerleider, 2002; Vuilleumier, Armony, Driver, \& Dolan, 2001 ), these findings suggest that the N170 reflects either earlier processing stages or, alternatively, that these fusiform activations by emotional contents are due to reentrant processing mechanisms (cf. Davis \& Whalen, 2001; Pessoa et al., 2002).

The first significant process of facilitated processing of threatening compared with neutral or friendly faces was augmented EPN amplitudes. This effect developed around $200 \mathrm{~ms}$ after stimulus onset and was maximally pronounced between 240 and $280 \mathrm{~ms}$. The topography of the EPN—bilaterally pronounced relative negativity over temporo-occipital areas-implicates visual processing areas. A similar topography of the EPN has been observed for emotional pictures taken from the IAPS (Lang et al., 1999) series (cf. Junghöfer et al., 2001). Rapid presentation of these pictures was found in a recent fMRI study to engage posterior visual processing areas (e.g., occipital gyrus, fusiform gyrus, STS; Junghöfer et al., 2002). Similarly, Kesler/West et al. (2001) observed increased activation of visual processing areas by anger compared with other facial expressions, in particular the fusiform gyrus and lateral occipital regions. According to these findings, one might assume a distributed network of visual processing areas as likely structures contributing to the generation of the EPN component. Overall, these findings are consistent with a scenario in which threatening stimuli are already tagged for further processing during sensory encoding, presumably on the basis of superficial stimulus analysis (cf. Öhman et al., 2000).

However, threatening expressions might also be subject to a continued elaborate stimulus analysis at later stages of information processing (cf. Öhman et al., 2000). Consistent with this hypothesis, a second process of facilitated processing of threatening faces was indicated by increased LPP amplitudes to threat compared with neutral or friendly faces. Again, as was the case for augmented EPN amplitudes, recent studies with emotional pictures observed enhanced LPP amplitudes (cf. Schupp et al., 2003b). A recent fMRI study with IAPS (Lang et al., 1999) pictures supports the assumption that the LPP might indicate more elaborate sustained perceptual processing
(Junghöfer et al., 2002). In this study, pictures were presented as a continuous stream of images, whereas the individual picture presentation time was either 333 $\mathrm{ms}$ or $1,500 \mathrm{~ms}$. If visual processing structures are implicated only in short-lived stimulus categorization, one would expect pronounced differences in blood oxygenation-level dependent signal change as a function of brief compared with long picture presentation times (see Pessoa et al., 2002, for a similar reasoning). By contrast, if visual processing structures are continuously engaged in stimulus processing, for example, by reentrant processes, one would expect little differences between both conditions. Interestingly, the distributed activation of visual processing areas by emotional pictures was similarly pronounced for emotional pictures of brief and more sustained presentations. Differences emerged only with regard to more anterior, prefrontal structures activated by emotional pictures during long presentations. Furthermore, recent ERP research established that the long picture presentation captures both processing components, whereas the fast picture presentation isolates the early facilitated processing (cf. Junghöfer et al., 2001; Schupp et al., 2003b). Taken together, although the functional significance of the augmented LPP amplitudes needs to be determined more directly in future studies, the present evidence is consistent with the hypothesis that the LPP reflects continued perceptual processing of threatening faces.

The facilitated processing of fear-relevant faces has been observed not only for threatening faces but also for fearful expressions. Specifically, a recent study by Sato et al. (2001) revealed increased EPN amplitudes to fear compared with neutral expressions. However, some inconsistencies across the present study and the Sato et al. study need to be acknowledged. For instance, Sato et al. did not observe enlarged LPP amplitudes to fearful expressions. Furthermore, they also observed enlarged EPN amplitudes to friendly compared with neutral expressions. At present, it remains unclear whether these differences are due to the emotional expression (threat vs. fear) or reflect methodological differences. For instance, with regard to the EPN modulation to friendly faces, Sato et al. included computer-morphed fear and friendly faces to increase emotional intensity. Furthermore, the subjects performed an explicit task by indicating the gender of the face. However, there is increasing evidence suggesting that the process indicated by the EPN occurs rather automatically and is independent of whether subjects are instructed to perform a concurrent explicit attention task. By contrast, the LPP appears to 
be more sensitive to conditions of resource competition (Ito \& Cacioppo, 2000; Schupp, Junghöfer, Weike, \& Hamm, 2003a). Thus, differences in stimulus materials and experimental task might have contributed to differing results.

The present data suggest that frontal presentations of the faces were associated with more reliable and consistent ERP modulations than lateral presentations. Nonetheless, all different orientations elicited substantial modulation of the EPN, whereas only the mid-left oriented faces appeared somewhat less potent to elicit threat-related augmented LPP amplitudes. Thus, the effects of facial orientation were not as pronounced as those revealed in aversive associative learning (Dimberg \& Öhman, 1983).

Overall, the data are consistent with the hypothesis of the facilitated processing of threatening faces. However, the subjective ratings indicate that threatening faces are perceived not only as more threatening and unpleasant compared with neutral or friendly faces but also as more arousing. These data raise the theoretical issue of whether the observed facilitated processing of threatening faces reflects primarily the threat-fear relevance or the increased arousal of the stimuli. Obviously, the increased arousal ratings associated with threatening faces bring the present data in line with previous findings of augmented EPN and LPP amplitudes to pleasant and unpleasant pictures, which were associated with increased arousal (Schupp et al., 2000, 2003b). Interestingly, facilitated perceptual processing in these studies was particularly pronounced for stimuli of high evolutionary significance, that is, images displaying erotic contents, mutilations, and threat (Schupp et al., in press, 2003b). Thus, these observations are consistent with the hypothesis that facilitated perceptual processing reflects evolutionary inheritance because evolutionary-relevant stimuli are particularly potent to activate primary appetitive or aversive motivational systems (with their associated pleasant and unpleasant affects; Lang, Bradley, \& Cuthbert, 1997; Öhman et al., 2000). In this conception, arousal is considered to reflect the intensity of either the appetitive or defensive motivational engagement, and not as a separate, independent dimension (Lang et al., 1997). Therefore, increased arousal ratings associated with threatening faces might be considered not as a confounding variable to be ruled out experimentally, but rather as reflecting the engagement of the defensive motivational system assuring the selective perceptual processing, attention capture, and rapid aversive learning observed for stimuli of evolutionary significance (cf. Öhman \& Mineka,
2001). Clearly, this reasoning is somewhat speculative and awaits future research. Varying threat, valence, and arousal of the stimulus materials, exploring social context demands, and manipulating the personal relevance of the expressions might help to further elucidate the phenomenon of facilitated perceptual processing of fear-relevant stimuli.

Interestingly, although the facilitated processing of threatening faces appears to be a consequence of intrinsic stimulus significance, similar ERP effects have been observed when selective processing of stimuli is brought out by explicit attention tasks (Näätänen, 1992). For instance, a temporo-occipital negativity is also observed when subjects are explicitly instructed to attend to particular stimulus features such as color or orientation (cf. Michie et al., 1999; Potts \& Tucker, 2001). Similarly, increased LPPs have been observed for attended stimuli calling for continued perceptual processing (cf. Ritter \& Ruchkin, 1992). These findings point to striking similarities in facilitated processing at the level of perceptual stimulus analysis because of attentional instruction or emotional significance. However, the facilitated processing of threatening faces presumably involves neural structures that are at least partially separate from those involved in the regulation of explicit attention. A widely held hypothesis suggests that a distributed neural network, including limbic structures, which is devoted to determine the significance of external stimuli, regulates the facilitated processing at the sensory cortical level (e.g., Davis \& Whalen, 2001; Derryberry \& Tucker, 1991; Pessoa et al., 2002). The amygdala might be one particularly relevant structure for rapid significance detection. The amygdala is responsive to emotional facial expression, and particularly to expressions of fear (e.g., Whalen et al., 2001), but enhanced regional blood flow in the amygdala has also been reported for threatening faces after aversive conditioning (Morris, Öhman, \& Dolan, 1998). In addition, Adolphs, Tranel, Damasio, and Damasio (1995) observed that a patient with bilateral damage of the amygdala was particularly impaired in the recognition of fear and anger expressions. Furthermore, amygdala activation does not depend on conscious identification of the stimuli because increased cerebral blood flow in the right amygdala has been observed to mask presentations of threatening faces (Morris, Öhman, \& Dolan, 1999). Such nonconscious activation of the amygdala appears to be occasioned through subcortical visual pathways, according to imaging data from a patient with visual blind sight (Morris, DeGelder, Weiskrantz, \& Dolan, 2001). Furthermore, the amyg- 
dala might facilitate processing in the visual cortex by direct projections, connections to anterior attention networks, or via ascending neuromodulatory systems (cf. Davis \& Whalen, 2001; Derryberry \& Tucker, 1991). However, the amygdala responds selectively not only to fear-relevant stimuli but also to other unpleasant and pleasant contents of high evolutionary significance. For instance, pictures of mutilations and erotica elicit amygdala activations (e.g., Hamann, Ely, Hoffman, \& Kilts, 2002). Furthermore, although a distributed network of rapid stimulus significance detection appears, in principle, well suited to regulate cortical stimulus processing, more direct evidence in support of this hypothesis is needed.

Recently, Öhman and Mineka (2001) summarized the evidence in favor of an evolved module of fear and fear learning in particular with respect to the selective and automatic activation by fear-relevant stimuli. It was suggested that automatic routines based on preexisting knowledge about the world might be used to detect significant cues in the environment. Such a mechanism in turn enables the tagging of stimuli for processing priority at a rather early stage of perceptual processing, which is presumably reflected by augmented EPN amplitudes to threatening faces. Continued processing ensures the transfer of visual information to working memory, and again, priority processing was observed for fear-relevant threatening faces at these later stages of information processing, as indicated by increased LPP amplitudes. These results might be a reflection of what Lang et al. (1997) described as natural selective attention to evolutionary significant stimuli in the environment.

\section{References}

Adolphs, R., Tranel, D., Damasio, H., \& Damasio, A. R. (1995). Fear and the human amygdala. Journal of Neuroscience, 15, 5879-5891.

Allison, T., Puce, A., \& McCarthy, G. (2000). Social perception from visual cues: Role of the STS region. Trends in Cognitive Sciences, 4, 267-278.

Bentin, S., Allison, T., Puce, A., Perez, E., \& McCarthy, G. (1996). Electrophysiological studies of face perception in humans. Journal of Cognitive Neuroscience, 8, 551-565.

Bradley, M. M., \& Lang, P. J. (1994). Measuring emotion: The Self-Assessment Manikin and the Sematic Differential. Journal of Behavioral Therapy \& Experimental Psychiatry, 25, 49-59.

Bradley, M. M., Sabatinelli, D., Lang, P. J., Fitzsimmons, J. R., King, W. M., \& Desai, P. (2003). Activation of the visual cortex in motivated attention. Behavioral Neuroscience, 2, 369-380.

Bruce, V., \& Young, A. W. (1986). Understanding face recognition. British Journal of Psychology, 77, 305-327.

Byrne, A., \& Eysenck, M. W. (1995). Trait anxiety, anxious mood, and threat detection. Cognition \& Emotion, 6, 549-562.

Cacioppo, J. T., Gardner, W. L., \& Berntson, G. G. (1999). The affect system has parallel and integrative processing components: Form follows function. Journal of Personality and Social Psychology, 76, 839-855.

Cauquil, A. S., Edmonds, G. E., \& Taylor, M. J. (2000). Is the face-sensitive N170 the only ERP not affected by selective attention? NeuroReport: For Rapid Communication of Neuroscience Research, 11, 2167-2171.

Cuthbert, B. N., Schupp, H. T., Bradley, M. M., Birbaumer, N., \& Lang, P. J. (2000). Brain potentials in affective picture processing: Covariation with autonomic arousal and affective report. Biological Psychology, 52, 95-111.

Davis, M., \& Whalen, P. J. (2001). The amygdala: Vigilance and emotion. Molecular Psychiatry, 6, 13-34.

Derryberry, D., \& Tucker, D. M. (1991). The adaptive base of the neural hierarchy: Elementary motivational controls on network function. In R. A. Dienstbier (Ed.), Nebraska Symposium on Motivation, 1990: Perspectives on motivation (pp. 289-342). Lincoln: University of Nebraska Press.

Dimberg, U., Elmehed, K., \& Thunberg, M. (2000). Unconscious facial reactions to emotional facial expressions. Psychological Science, 11, 86-89.

Dimberg, U., \& Öhman, A. (1983). The effects of directional facial cues on electrodermal conditioning to facial stimuli. Psychophysiology, 20, 160-167.

Dimberg, U., \& Thunberg, M. (1998). Rapid facial reactions to emotional facial expressions. Scandinavian Journal of Psychology, 39, 39-45.

Ekman, P., \& Friesen, W. V. (1975). Unmasking the face. Englewood Cliffs, NJ: Prentice Hall.

Esteves, F., Dimberg, U., \& Öhman, A. (1994). Automatically elicited fear: Conditioned skin conductance responses to masked facial expressions. Cognition \& Emotion, 8, 393-413.

Esteves, F., Parra, C., Dimberg, U., \& Öhman, A. (1994). Nonconscious associative learning: Pavlovian conditioning of skin conductance responses to masked fearrelevant facial stimuli. Psychophysiology, 31, 375-385.

Folk, C. L., Remington, R. W., \& Johnston, J. C. (1992). Involuntary covert orienting is contingent on attentional control settings. Journal of Experimental Psychology: Human Perception and Performance, 18, 1030-1044.

Fox, E., Lester, V., Russo, R., Bowles, R. J., Pichler, A., \& Dutton, K. (2000). Facial expressions of emotion: Are 
angry faces detected more efficiently? Cognition \& Emotion, 14, 61-92.

Fredrikson, M., Wik, G., Greitz, T., Eriksson, L., Stone, E. S., Ericson, K., \& Sedvall, G. (1993). Regional cerebral blood flow during experimental phobic fear. Psychophysiology, 30, 126-130.

Gilboa-Schechtman, S. E., Foa, E. B., \& Amir, N. (1999). Attentional biases for facial expressions in social phobia: The face-in-the-crowd paradigm. Cognition \& Emotion, 13, 305-318.

Hamann, S. B., Ely, T. D., Hoffman, J. M., \& Kilts, C. D. (2002). Ecstasy and agony: Activation of the human amygdala in positive and negative emotion. Psychological Science, 13, 135-141.

Haxby, J. V., Hoffman, E. A., \& Gobbini, M. I. (2000). The distributed human neural system for face perception. Trends in Cognitive Sciences, 4, 223-233.

Holmes, A., Vuilleumier, P., \& Eimer, M. (2003). The processing of emotional facial expression is gated by spatial attention: Evidence from event-related brain potentials. Cognitive Brain Research, 16, 174-184.

Ito, T., \& Cacioppo, J. T. (2000). Electrophysiological evidence of implicit and explicit categorization processes. Journal of Experimental Social Psychology, 36, 660-676.

Johnsen, B. H., Thayer, J. F., \& Hugdahl, K. (1995). Affective judgment of the Ekman faces: A dimensional approach. Journal of Psychophysiology, 9, 193-202.

Junghöfer, M., Bradley, M. M., Elbert, T. R., \& Lang, P. J. (2001). Fleeting images: A new look at early emotion discrimination. Psychophysiology, 38, 175-178.

Junghöfer, M., Schupp, H. T., Stark, R., Schienle, A., Elbert, T., Hamm, A. O., \& Vaitl, D. (2002). Valence, arousal and selective picture processing: An fMRI analysis [Abstract]. Psychophysiology, 39, S14-S15.

Kesler/West, M. L., Andersen, A. H., Smith, C. D., Avison, M. J., Davis, C. E., Kryscio, R. J., \& Blonder, L. X. (2001). Neural substrates of facial emotion processing using fMRI. Cognitive Brain Research, 11, 213-226.

Lang, P. J. (1980). Behavioral treatment and the biobehavioral assessment: Computer applications. In J. B. Sidowski, J. H. Johnson, \& T. A. Williams (Eds.), Technology in mental health care delivery systems (pp. 119137). Norwood, NJ: Ablex Publishing.

Lang, P. J., Bradley, M. M., \& Cuthbert, B. N. (1997). Motivated attention: Affect, activation, and action. In P. J. Lang, R. F. Simons, \& M. Balaban (Eds.), Attention and orienting: Sensory and motivational processes (pp. 97135). Mahwah, NJ: Erlbaum.

Lang, P. J., Bradley, M. M., \& Cuthbert, B. N. (1999). International Affective Picture System (IAPS): Instruction manual and affective ratings (Tech Rep. No. A-4).
Gainesville: University of Florida, The Center for Research in Psychophysiology.

Luck, S. J., Woodman, G. F., \& Vogel, E. K. (2000). Eventrelated potential studies of attention. Trends in Cognitive Science, 4, 432-440.

Lundqvist, D., Esteves, F., \& Öhman, A. (1999). The face of wrath: Critical features for conveying facial threat. Cognition \& Emotion, 13, 691-711.

Lundqvist, D., Flykt, A., \& Öhman, A. (1998). The Karolinska Directed Emotional Faces. Stockholm: Karolinska Institute, Psychology section, Department of Clinical Neuroscience.

Mangun, G. R., \& Hillyard, S. A. (1991). Mechanisms and models of selective attention. In M. D. Rugg \& M. G. H. Coles (Eds.), Electrophysiology of mind (pp. 40-85). Oxford, England: Oxford University Press.

Michie, P. T., Karayanidis, F., Smith, G. L., Barrett, N. A., Large, M. M., O'Sullivan, B. T., \& Kavangh, D. J. (1999). An exploration of varieties of visual attention: ERP findings. Cognitive Brain Research, 7, 419-450.

Mogg, K., Millar, N., \& Bradley, B. P. (2000). Biases in eye movements to threatening facial expressions in generalized anxiety disorder and depressive disorder. Journal of Abnormal Psychology, 109, 695-704.

Morris, J. S., DeGelder, B., Weiskrantz, L., \& Dolan, R. J. (2001). Differential extrageniculostriate and amygdala responses to presentation of emotional faces in a cortically blind field. Brain, 124, 1241-1252.

Morris, J. S., Öhman, A., \& Dolan, R. J. (1998, June). Conscious and unconscious emotional learning in the human amygdala. Nature, 393, 467-470.

Morris, J. S., Öhman, A., \& Dolan, R. J. (1999). A subcortical pathway to the right amygdala mediating "unseen" fear. Proceedings of the National Academy of Sciences, USA, 96, 1680-1685.

Näätänen, R. (1992). Attention and brain function. Hillsdale, NJ: Erlbaum.

Öhman, A. (1986). Face the beast and fear the face: Animal and social fears as prototypes for evolutionary analyses of emotion. Psychophysiology, 23, 123-145.

Öhman, A., \& Dimberg, U. (1978). Facial expressions as conditioned stimuli for electrodermal responses: A case of "preparedness"? Journal of Personality and Social Psychology, 36, 1251-1258.

Öhman, A., Flykt, A., \& Esteves, F. (2001). Emotion drives attention: Detecting the snake in the grass. Journal of Experimental Psychology: General, 130, 466-478.

Öhman, A., Flykt, A., \& Lundqvist, D. (2000). Unconscious emotion: Evolutionary perspectives, psychophysiological data and neuropsychological mechanisms. In R. D. Lane \& L. Nadel (Eds.), Cognitive neuroscience of emotion (pp. 296-327). New York: Oxford University Press. 
Öhman, A., Lundqvist, D., \& Esteves, F. (2001). The face in the crowd revisited: A threat advantage with schematic stimuli. Journal of Personality and Social Psychology, 80, 381-396.

Öhman, A., \& Mineka, S. (2001). Fears, phobias, and preparedness: Toward an evolved module of fear and fear learning. Psychological Review, 108, 483-522.

Pessoa, L., Kastner, S., \& Ungerleider, L. G. (2002). Attentional control of the processing of neutral and emotional stimuli. Cognitive Brain Research, 15, 31-45.

Potts, G. F., \& Tucker, D. M. (2001). Frontal evaluation and posterior representation in target detection. Cognitive Brain Research, 11, 147-156.

Ritter, W., \& Ruchkin, D. S. (1992). A review of eventrelated potential components discovered in the context of studying P3. In D. Friedman \& G. E. Bruder (Eds.), Psychophysiology and experimental psychopathology: $A$ tribute to Samuel Sutton (pp. 1-32). New York: New York Academy of Sciences.

Sato, W., Kochiyama, T., Yoshikawa, S., \& Matsumura, M. (2001). Emotional expression boosts early visual processing of the face: ERP recording and its decomposition by independent component analysis. Neuroreport, 12, 709714.

Schupp, H. T., Cuthbert, B. N., Bradley, M. M., Cacioppo, J. T., Ito, T., \& Lang, P. J. (2000). Affective picture processing: The late positive potential is modulated by motivational relevance. Psychophysiology, 37, 257-261.
Schupp, H. T., Cuthbert, B. N., Bradley, M. M., Hillman, C. H., Hamm, A. O., \& Lang, P. J. (in press). Brain processes in emotional perception: Motivated attention. Cognition \& Emotion.

Schupp, H. T., Junghöfer, M., Weike, A., \& Hamm, A. (2003a). Attention and emotion: An ERP analysis of facilitated emotional stimulus processing. NeuroReport, 14, 1107-1110.

Schupp, H. T., Junghöfer, M., Weike, A., \& Hamm, A. (2003b). Emotional facilitation of sensory processing in the visual cortex. Psychological Science, 14, 7-13.

Tipples, J., Atkinson, A. P., \& Young, A. W. (2002). The eyebrow frown: A salient social signal. Emotion, 2, 288296.

Vuilleumier, P., Armony, J. L., Driver, J., \& Dolan, R. J. (2001). Effects of attention and emotion on face processing in the human brain: An event-related fMRI study. Neuron, 30, 829-841.

Whalen, P. J., Shin, L. M., McInerney, S. C., Fischer, H., Wright, C. I., \& Rauch, S. L. (2001). A functional MRI study of human amygdala responses to facial expressions of fear versus anger. Emotion, 1, 70-83.

Received December 16, 2002

Revision received October 23, 2003

Accepted October 24, 2003 